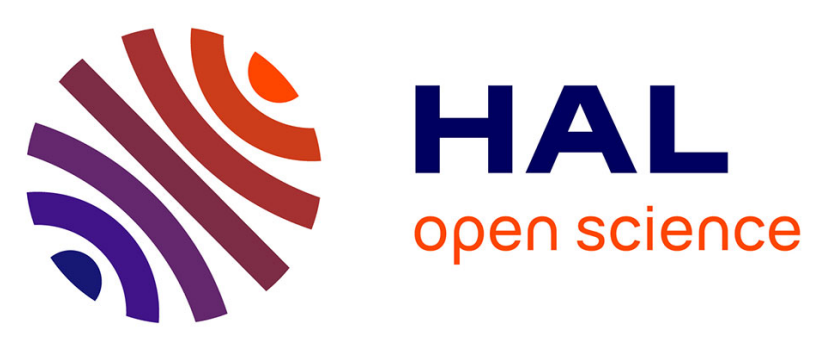

\title{
Control of a class of nonlinear cascade systems with input-dependent saturations
}

\author{
Céline Casenave, Marc Perez
}

\section{To cite this version:}

Céline Casenave, Marc Perez. Control of a class of nonlinear cascade systems with input-dependent saturations. 27. Chinese Control and Decision Conference (CCDC ), Institute of Electrical and Electronics Engineers (IEEE). Singapour, USA., May 2015, Qingdao, China. hal-01154868v2

\section{HAL Id: hal-01154868 \\ https://hal.science/hal-01154868v2}

Submitted on 23 Oct 2018

HAL is a multi-disciplinary open access archive for the deposit and dissemination of scientific research documents, whether they are published or not. The documents may come from teaching and research institutions in France or abroad, or from public or private research centers.
L'archive ouverte pluridisciplinaire $\mathbf{H A L}$, est destinée au dépôt et à la diffusion de documents scientifiques de niveau recherche, publiés ou non, émanant des établissements d'enseignement et de recherche français ou étrangers, des laboratoires publics ou privés. 


\title{
Control of a class of nonlinear cascade systems with input-dependent saturations
}

\author{
Céline Casenave ${ }^{1}$, Marc Perez $^{2}$ \\ 1. INRA UMR INRA-SupAgro MISTEA, Montpellier 34060, France \\ E-mail: celine.casenave@supagro.inra.fr \\ 2. INRA UMR SPO, Montpellier 34060, France \\ E-mail: marc.perez@supagro.inra.fr
}

\begin{abstract}
In the present paper, we propose a control strategy for the stabilization of a class of nonlinear cascade systems with input-dependent saturation. The saturation constraint considered is a constraint of order: the control input of one subsystem has to be smaller than the control input of the preceding subsystem of the cascade structure. This class of systems include interconnected reactors device such as multi-stage continuous fermentors. The control law is designed on the basis of a specific Lyapunov function previously introduced by R.Antonelli and A.Astolfi in [1]. It guarantees the stability of the closed-loop system, the convergence of the state variable to the set-point and the fulfillment of the saturation constraint all along the trajectories of the closed-loop system. The approach is applied, in numerical simulation, to a concrete example of a series of interconnected continuous bioreactors.
\end{abstract}

Key Words: cascade systems; nonlinear system; input-dependent saturation; Lyapunov function; control design

\section{INTRODUCTION}

When working on real processes, whether they are physical, chemical or biological, there are often some technical constraints that we have to take into account for the design of the control strategies. One classical constraint is the saturation of the inputs, which often refers to the physical limitation of the actuators. Such kind of constraint has been widely studied in the literature for linear systems, and several approaches have been developed in that case. Some of these approaches try to compensate the effect of the saturation on the behavior of the closed-loop system, as the anti-windup techniques [2]; other ones directly include the saturation in the initial model before designing the control law as it is the case, for example, of methods based on polytopic representation of the saturation [3]. However, in the case of nonlinear systems, the problem is more complex which explains why few research works have been published for the moment. In these works, the authors generally try to adapt control strategies initially developed for linear systems to nonlinear ones. It has lead to some interesting contributions about feedback linearization control for nonlinear systems with input constraints $[4,5,6,7]$. However, input-output linearization is not always possible and can be less efficient than other more advanced control strategies. So, what can be done in that case? One alternative can be found in the really interesting paper written by R.Antonelli and A.Astolfi whose title is "Continuous stirred tank reactors: easy to stabilize?" [1]. In this paper, the authors propose a control strat- egy to stabilize CSTR, which is based on a specific Lyapunov function in which the bounds of the control inputs explicitly appear. The control law designed on the basis of this Lyapunov function, guarantees the stability of the closed-loop system, the convergence of the state variable to the set-point and the fulfillment of the saturation constraint all along the trajectories of the closed-loop system. The only required hypothesis is that the set-point has to be the single and globally asymptotically stable equilibrium point of the system for a given constant value of the input, which fulfills the saturation constraint. But, as always with Lyapunov based control, a Lyapunov function has also to be known, which is the main difficulty of the approach. Even if the paper of Antonelli and Astolfi is devoted to the control of CSTR, the approach used to design the control law is general and can be applied to a wide class of systems, for both stabilization [8] or design of adaptive control laws [9].

In the present paper, we will extend the approach presented in [1] to a class of nonlinear cascade systems with input dependent saturations. The saturation constraint considered is a constraint of order: the control input of one subsystem has to be smaller than the control input of the preceding one (in the cascade structure). This class of systems include interconnected continuous reactors device such as multi-stage continuous fermentor (MSCF) [10,11]. Indeed, the input flow rates of each reactor of the MSCF, which are taken as control inputs, are constrained, the input flow rate of one reactor being necessarily smaller than the output 
flow rate of the preceding one.

The paper is organized as follows. In section 2, we first recall the result presented in [1] and propose an extension to a class of multi-input nonlinear systems. Then, the case of cascade systems with input saturation constraint of order type is considered. In section 3 , the control strategy is applied on a concrete example of a series of interconnected continuous bioreactors. Some numerical simulations are given to highlight the efficiency of the method.

\section{CONTROL of CASCADE SYSTEMS with INPUT SATURATION CON- STRAINT of ORDER TYPE}

In this section, we propose a control strategy for the stabilization of a class of nonlinear cascade systems with input-dependent saturation of order type. This control strategy is based on an extension of a result presented in [1], which is recalled here-after.

Lemma 1 [1] Consider the system

$$
\frac{d \xi}{d t}=\phi(\xi)+\psi(\xi) v
$$

where $\xi(t) \in \mathbb{R}^{n}$ is the state, $v(t) \in \mathbb{R}$ is the control, and $\phi, \psi: \mathbb{R}^{n} \mapsto \mathbb{R}^{n}$. Suppose that there exist a constant control $v=v^{*}$ and a positive definite and radially unbounded function $V(\xi)$ such that:

$$
\begin{aligned}
\phi(0)+\psi(0) v^{*} & =0, \\
\forall \xi \neq 0, \frac{d V}{d t}=\frac{\partial V}{\partial \xi} \phi(\xi)+\frac{\partial V}{\partial \xi} \psi(\xi) v^{*} & <0 .
\end{aligned}
$$

Then, for any $\underline{v}$ and $\bar{v}$ such that $\underline{v}<v^{*}<\bar{v}$, there exists a dynamic control law, which does not require the knowledge of $v^{*}$, namely $v=\theta$ with:

$$
\frac{d \theta}{d t}=k \frac{\partial V}{\partial \xi} \psi(\xi)(\theta-\bar{v})(\theta-\underline{v}),
$$

with $\theta(0) \in(\underline{v}, \bar{v})$ and $k>0$, such that the closed loop system (1)-(4) is stable in the sense of Lyapunov, the variable $\xi$ converges to zero whatever the initial condition $\xi(0)$ and, along the trajectories of the closed loop system, $\theta(t)=v(t) \in(\underline{v}, \bar{v}), \forall t>0$.

The result presented in lemma 1 is suitable for nonlinear input-affine systems with constant input saturation. In lemma 2, we present an extension of this result to a class of multi-input nonlinear systems.

Lemma 2 Consider the system

$$
\frac{d \xi_{j}}{d t}=\phi_{j}(\xi)+\psi_{j}(\xi) \prod_{k=1}^{j} v_{k}, j=1: n
$$

where $\xi=\left(\xi_{1}^{T}, \ldots, \xi_{n}^{T}\right)^{T}$ with $\xi_{j}(t) \in \mathbb{R}^{n_{j}}$ is the state, $v_{j}(t) \in \mathbb{R}, j=1: n$ are the control inputs, and $\phi_{j}, \psi_{j}$ : $\mathbb{R}^{N} \mapsto \mathbb{R}^{n_{j}}$ with $N=\sum_{k=1}^{n} n_{k}$. Let $\Omega$ be an invariant set of (5) to which belongs 0 . Suppose that there exist some constant control inputs $v_{j}=v_{j}^{*}, j=1: n$, and a function $V(\xi)$, positive definite in $\Omega$ and such that:

$$
\begin{array}{r}
\forall j=1: n, \phi_{j}(0)+\psi_{j}(0) \prod_{k=1}^{j} v_{k}^{*}=0 ; \\
\frac{d V}{d t}=\sum_{j=1}^{n} \frac{\partial V}{\partial \xi_{j}}\left[\phi_{j}(\xi)+\psi_{j}(\xi) \prod_{k=1}^{j} v_{k}^{*}\right] \leqslant 0 \text { in } \Omega ; \\
\text { and }\left\{\xi \in \Omega \text { such that } \frac{d V}{d t}=0\right\}=\{0\} .
\end{array}
$$

Then, for any $v_{j}$ and $\overline{v_{j}}$ such that $v_{j}<v_{j}^{*}<\overline{v_{j}}$, there exists a dynamic control law, which does not require the knowledge of $v_{j}^{*}$, namely $v_{j}=\theta_{j}$ with $\sigma_{j}\left(\theta_{j}\right)=$ $k_{j}\left(\theta_{j}-\overline{v_{j}}\right)\left(\theta_{j}-\underline{v_{j}}\right)$ and:

$$
\begin{aligned}
& \frac{d \theta_{n}}{d t}=\sigma_{n}\left(\theta_{n}\right) \frac{\partial V}{\partial \xi_{n}} \psi_{n}(\xi) \\
& \frac{d \theta_{j \neq n}}{d t}=\sigma_{j}\left(\theta_{j}\right)\left[\frac{\partial V}{\partial \xi_{j}} \psi_{j}(\xi)+\sum_{l=j+1}^{n} \frac{\partial V}{\partial \xi_{l}} \psi_{l}(\xi) \prod_{k=j+1}^{l} \theta_{k}\right]
\end{aligned}
$$

with $\theta_{j}(0) \in\left(v_{j}, \overline{v_{j}}\right)$ and $k_{j}>0$, such that the closed loop system (5)-(9) is asymptotically stable in the sense of Lyapunov, the variable $\xi$ converges to 0 whatever the initial condition $\xi(0) \in \Omega$ and, along the trajectories of the closed loop system, $\theta_{j}(t)=v_{j}(t) \in$ $\left(\underline{v_{j}}, \overline{v_{j}}\right), \forall t>0$.

Proof. Consider the function $W(\xi, \theta)=V(\xi)+$ $\sum_{j=1}^{n} \prod_{k=0}^{j-1} v_{k}^{*}\left(\omega_{j}\left(\theta_{j}\right)-\omega_{j}\left(v_{j}^{*}\right)\right)$ with $v_{0}^{*}=1$, and where:

$$
\omega_{j}(\theta)=\ln \left(\left(\overline{v_{j}}-\theta_{j}\right)^{\tau_{j} / k_{j}}\right)-\ln \left(\left(\theta_{j}-\underline{v_{j}}\right)^{\left(\tau_{j}+1\right) / k_{j}}\right)
$$

with $k_{j}>0$ and $\tau_{j}=\frac{v_{j}^{*}-\overline{v_{j}}}{\overline{v_{j}}-\underline{v_{j}}}<0$.

Note that $\tau_{j}+1=\frac{v_{j}^{*}-v_{j}}{\overline{v_{j}}-v_{j}}>0$. Moreover, the function $W$ is positive in $\Omega_{\xi \theta}=$ $\left\{(\xi, \theta) \in \Omega \times \mathbb{R}^{n} \mid \underline{v_{j}}<\theta_{j}<\overline{v_{j}}, \forall j=1: n\right\}, \quad$ and such that $W(\xi, \theta)=0$ if and only if $\xi=0$ and $\theta_{j}=v_{j}^{*}, \forall j=1: n$. Indeed we have:

- $V$ is positive definite in $\Omega$ and for all $j=1: n$, the function $\omega_{j}$ is such that:

$$
\begin{gathered}
\forall \theta_{j} \in \mathbb{R}, \quad \frac{\partial \omega_{j}}{\partial \theta_{j}}=\frac{1}{k_{j}} \frac{\theta_{j}-v_{j}^{*}}{\left(\overline{v_{j}}-\theta_{j}\right)\left(\theta_{j}-\underline{v_{j}}\right)} \\
\begin{cases}<0 & \text { if } v_{j}<\theta_{j}<v_{j}^{*}<\overline{v_{j}} \\
=0 & \text { if } \overline{v_{j}}<\theta_{j}=v_{j}^{*}<\overline{v_{j}} \\
>0 & \text { if } \underline{v_{j}}<v_{j}^{*}<\theta<\overline{v_{j}} .\end{cases}
\end{gathered}
$$

- So we have, $\forall \theta_{j} \in\left(v_{j}, \overline{v_{j}}\right) \backslash\left\{v_{j}^{*}\right\}, \omega_{j}\left(\theta_{j}\right)-\omega_{j}\left(v_{j}^{*}\right)>0$, and $W(\xi, \theta)=0 \Leftrightarrow \overline{V(\xi)}=0$ and $\omega_{j}\left(\theta_{j}\right)=\omega_{j}\left(v_{j}^{*}\right), \forall j$ $\Leftrightarrow \xi=0$ and $\theta_{j}=v_{j}^{*}, \forall j$. 
Consequently (with $v_{j}=\theta_{j}$, and the control law defined by (9)):

$$
\begin{aligned}
& \frac{d W}{d t}=\sum_{j=1}^{n}\left(\frac{d \xi_{j}}{d t} \frac{\partial W}{\partial \xi_{j}}+\frac{d \theta_{j}}{d t} \frac{\partial W}{\partial \theta_{j}}\right) \\
& =\sum_{j=1}^{n}\left(\phi_{j}(\xi)+\psi_{j}(\xi) \prod_{p=1}^{j} v_{p}^{*}\right) \frac{\partial V}{\partial \xi_{j}} \\
& +\underbrace{\left[\prod_{k=1}^{1} \theta_{k}-\theta_{1} \prod_{p=0}^{1-1} v_{p}^{*}\right]}_{=0} \frac{\partial V}{\partial \xi_{1}} \psi_{1}(\xi)+\sum_{j=2}^{n} A_{j}\left(\theta, v^{*}\right) \frac{\partial V}{\partial \xi_{j}} \psi_{j}(\xi)
\end{aligned}
$$

with, $\forall j=2: n, A_{j}\left(\theta, v^{*}\right):=\prod_{k=1}^{j} \theta_{k}-\theta_{j} \prod_{p=0}^{j-1} v_{p}^{*}$

$$
+\sum_{l=1}^{j-1}\left[\prod_{k=l+1}^{j} \theta_{k}\left(v_{l}^{*}-\theta_{l}\right) \prod_{p=0}^{l-1} v_{p}^{*}\right]
$$

We will now show, by recurrence, that, $\forall j=2$ : $n, A_{j}\left(\theta, v^{*}\right)=0$. First, for $j=2$, we have:

$$
\begin{aligned}
A_{2}\left(\theta, v^{*}\right) & =\theta_{1} \theta_{2}-\theta_{2} v_{1}^{*}+\prod_{k=2}^{2} \theta_{k}\left(v_{1}^{*}-\theta_{1}\right) \prod_{p=0}^{0} v_{p}^{*} \\
& =\theta\left[\theta_{1}-v_{1}^{*}+\left(v_{1}^{*}-\theta_{1}\right) v_{0}^{*}\right] \\
& \left.=0 \text { (because } v_{0}^{*}=1\right) .
\end{aligned}
$$

Then, suppose that $A_{j-1}\left(\theta, v^{*}\right)=0$; we have:

$$
\begin{aligned}
& A_{j}\left(\theta, v^{*}\right)=\prod_{k=1}^{j} \theta_{k}-\theta_{j} \prod_{p=0}^{j-1} v_{p}^{*}+\theta_{j}\left(v_{j-1}^{*}-\theta_{j-1}\right) \prod_{p=0}^{j-2} v_{p}^{*} \\
& +\sum_{l=1}^{j-2}\left[\prod_{k=l+1}^{j} \theta_{k}\left(v_{l}^{*}-\theta_{l}\right) \prod_{p=0}^{l-1} v_{p}^{*}\right] \\
& =\theta_{j}(\prod_{k=1}^{j-1} \theta_{k}-\underbrace{\prod_{p=0}^{j-1} v_{p}^{*}+v_{j-1}^{*} \prod_{p=0}^{j-2} v_{p}^{*}}_{=0}-\theta_{j-1} \prod_{p=0}^{j-2} v_{p}^{*}) \\
& +\theta_{j} \sum_{l=1}^{j-2}\left[\prod_{k=l+1}^{j-1} \theta_{k}\left(v_{l}^{*}-\theta_{l}\right) \prod_{p=0}^{l-1} v_{p}^{*}\right]=\theta_{j} A_{j-1}\left(\theta, v^{*}\right)=0 .
\end{aligned}
$$

So, finally, $\frac{d W}{d t}=\sum_{j=1}^{n}\left(\phi_{j}(\xi)+\psi_{j}(\xi) \prod_{p=1}^{j} v_{p}^{*}\right) \frac{\partial V}{\partial \xi_{j}} \leqslant 0$ in $\Omega$. As $\left\{\xi \in \Omega\right.$ such that $\left.\frac{d V}{d t}=0\right\}=\{0\}$, we obtain the first part of the lemma by application of the Lasalle's invariance theorem. By definition of the control law dynamics $(9)$, and because $\theta_{j}(0) \in$ $\left(\underline{v}_{j}, \bar{v}_{j}\right), \forall j=1: n$, we have $\theta_{j}(t)=v_{j}(t) \in\left(\underline{v}_{j}, \bar{v}_{j}\right)$ for all $j=1: n$ and for all $t>0$.

Remark 1 Note that, contrary to lemma 1, the function $V$ is not assumed to be radially unbounded in lemma 2 and an invariant set $\Omega$ has been introduced. This choice was made because of the example of application presented in this paper. However, as for all Lyapunov based control techniques, variations on this result can easily be formulated.
Let us now consider a class of nonlinear cascade systems, composed of $n$ sub-systems connected in series, that is some systems of the form:

$$
\frac{d x_{j}}{d t}=f_{j}\left(x_{j}, x_{j-1}\right)+g_{j}\left(x_{j}, x_{j-1}\right) u_{j}, j=1: n
$$

with, for all $j=1: n, x_{j} \in \mathbb{R}^{n_{j}}, n_{j} \in \mathbb{R}, u_{j} \in \mathbb{R}$, and $f_{j}, g_{j}: \mathbb{R}^{j} \times \mathbb{R}^{j-1} \rightarrow \mathbb{R}^{j}$. We assume that the inputs of the sub-systems are constrained in the following way:

$$
\underline{u}<u_{n}<u_{n-1}<\ldots<u_{1}<\bar{u},
$$

with $\underline{u}, \bar{u} \in \mathbb{R}$. An example of such a system is given in section 3 .

Proposition 1 Consider the system (11). Let denote $x=\left(x_{1}^{T}, \ldots, x_{n}^{T}\right)^{T}, u=\left(u_{1}, \ldots, u_{n}\right)^{T}$ and $\Omega$ an invariant set of (11) to which belongs 0 . Suppose that there exist a constant control input $u^{*}=\left(u_{1}^{*}, \ldots, u_{n}^{*}\right)^{T}$ which fulfills the constraint (12), and a function $V(x)$, positive definite in $\Omega$ such that:

$$
\begin{array}{r}
\forall j=1: n, f_{j}(0,0)+g_{j}(0,0) u_{j}^{*}=0 ; \\
\frac{d V}{d t}=\sum_{j=1}^{n} \frac{\partial V}{\partial x_{j}}\left[f_{j}\left(x_{j}, x_{j-1}\right)+g_{j}\left(x_{j}, x_{j-1}\right) u_{j}^{*}\right] \leqslant 0 \text { in } \Omega ; \\
\text { and }\left\{x \in \Omega \text { such that } \frac{d V}{d t}=0\right\}=\{0\},
\end{array}
$$

$$
\begin{aligned}
\text { with } \phi_{j}(x) & =f_{j}\left(x_{j}, x_{j-1}\right)+g_{j}\left(x_{j}, x_{j-1}\right) \underline{u}, \\
\psi_{j}(x) & =g_{j}\left(x_{j}, x_{j-1}\right)(\bar{u}-\underline{u}) .
\end{aligned}
$$

Then, there exists a dynamic control law, which does not require the knowledge of $u_{j}^{*}$, namely $u_{j}=(\bar{u}-$ $\underline{u}) \prod_{k=1}^{j} \theta_{k}+\underline{u}$ with $\sigma_{j}\left(\theta_{j}\right)=k_{j}\left(\theta_{j}-1\right) \theta_{j}$ and

$$
\begin{aligned}
& \frac{d \theta_{n}}{d t}=\sigma_{n}\left(\theta_{n}\right) \frac{\partial V}{\partial x_{n}} \psi_{n}(x), \\
& \frac{d \theta_{j \neq n}}{d t}=\sigma_{j}\left(\theta_{j}\right)\left[\frac{\partial V}{\partial x_{j}} \psi_{j}(x)+\sum_{l=j+1}^{n} \frac{\partial V}{\partial x_{l}} \psi_{l}(x) \prod_{k=j+1}^{l} \theta_{k}\right],
\end{aligned}
$$

with $\theta_{j}(0) \in(0,1)$ and $k_{j}>0$, such that the closed loop system (11)-(15) is asymptotically stable in the sense of Lyapunov, the variable $x$ converges to 0 whatever the initial condition $x(0) \in \Omega$ and, along the trajectories of the closed loop system, $u(t)$ fulfills the constraint (12) $\forall t>0$.

Proof. The result of proposition 1 is obtained by application of lemma 2 to the cascade system (11), after the following change of variables:

$$
v_{1}=\frac{u_{1}-\underline{u}}{\bar{u}-\underline{u}} ; v_{j}=\frac{u_{j}-\underline{u}}{u_{j-1}-\underline{u}}, \forall j=2: n,
$$

which leads to $u_{j}=(\bar{u}-\underline{u}) \prod_{k=1}^{j} v_{k}+\underline{u}, \forall j=1: n$. 


\section{EXAMPLE OF APPLICATION}

\subsection{Model of the system}

Consider a system composed of $n$ reactors connected in series, in which some micro-organisms $X$ (bacteria, yeasts, etc.) grow on a given substrate $S$, with a yield coefficient $k$ :

$$
S \longrightarrow k X \text {. }
$$

The output flow rate of each reactor is equal to its input flow rate, in such way that the volumes $\nu_{j}, j=$ $1: n$ of the reactors remain constant. The first reactor is fed with a solution only containing some substrate. The other reactors are fed from the output of the previous one without any external addition. The input flow rate $Q_{j}, j=1: n$ of the reactors are controlled independently by some piston pumps, the only constraint (coming from the cascade structure of the device) being that the input flow rate $Q_{j}$ of the $j^{\text {th }}$ reactor has to be lower than the output flow rate $Q_{j-1}$ of the $(j-1)^{\text {th }}$ reactor:

$$
Q_{m}<Q_{n}<Q_{n-1}<\ldots<Q_{2}<Q_{1}<Q_{M},
$$

with $Q_{m}$ and $Q_{M}$ the minimal and maximal flow rates which can be applied. An example of such kind of system can be found in $[10,11]$.

The classical model for such a system is given by, $\forall j=$ $1: n$ :

$$
\begin{aligned}
\frac{d X_{j}}{d t} & =\mu\left(S_{j}\right) X_{j}+D_{j}\left(X_{j-1}-X_{j}\right) \\
\frac{d S_{j}}{d t} & =-k \mu\left(S_{j}\right) X_{j}+D_{j}\left(S_{j-1}-S_{j}\right),
\end{aligned}
$$

with $X_{j}$ and $S_{j}$ the biomass and substrate concentrations in the $j^{\text {th }}$ reactor, $X_{0}(=0)$ and $S_{0}$ the biomass and substrate concentrations of the medium feeding the first reactor, $D_{j}=\frac{Q_{j}}{\nu_{j}}$ the dilution rate of the $j^{\text {th }}$ reactor, $k$ the yield coefficient, and $\mu: S \mapsto \mu(S)$ the growth function of the micro-organisms that we assume to be increasing on $\mathbb{R}^{+}$, and more specifically of the Monod form, that is:

$$
\mu(S)=\frac{m S}{a+S}, \text { with } m, a>0 .
$$

We assume that, before applying the control law, the system is at equilibrium, and we denote $Q_{j}^{0}$ the initial constant input values which are such that:

$$
Q_{m}<Q_{n}^{0}<Q_{n-1}^{0}<\cdots<Q_{2}^{0}<Q_{1}^{0}<Q_{M} .
$$

If we look at the total amount of matter $k X_{j}+S_{j}$ in the $j^{\text {th }}$ reactor, we note that:

$$
k \frac{d X_{j}}{d t}+\frac{d S_{j}}{d t}=D_{j}\left[\left(k X_{j-1}+S_{j-1}\right)-\left(k X_{j}+S_{j}\right)\right] .
$$

At equilibrium, we therefore have $\forall j, k X_{j}^{*}+S_{j}^{*}=$ $k X_{0}+S_{0}\left(=S_{0}\right)$. Moreover, if $k X_{j}(0)+S_{j}(0)=S_{0}, \forall j$, then, $\forall t>0, \forall j, k X_{j}(t)+S_{j}(t)=S_{0}$ and system (1920) can be simplified in: $\forall j=1: n, \forall t>0$,

$$
\frac{d S_{j}}{d t}=-\mu\left(S_{j}\right)\left(S_{0}-S_{j}\right)+D_{j}\left(S_{j-1}-S_{j}\right) .
$$

\subsection{Stability analysis}

System (24) has several properties which will be given in the sequel without being proved, for lack of place. First, we can show that

$$
\Omega:=\{S \in] 0, S_{0}\left[^{n}, \text { such that } S_{j} \leqslant S_{j-1}\right\}
$$

is a positive invariant set for $(24)$.

Because $\mu$ is an increasing function, we can also show that, for any values $\left\{Q_{j}^{*}\right\}_{j=1: n}$ of $\left\{Q_{j}\right\}_{j=1: n}$ which fulfills the constraint (18), there exists a unique equilibrium point $S^{*}=\left(S_{1}^{*}, \ldots S_{n}^{*}\right)^{T} \in \Omega$.

Let us now consider the functions $\left.V_{j}:\right] 0, S_{0}\left[\mapsto \mathbb{R}^{+}, j=\right.$ $1: n$, defined as follows:

$$
\begin{aligned}
V_{1}\left(S_{1}\right) & =\int_{S_{1}^{*}}^{S_{1}} \frac{s-S_{1}^{*}}{\left(S_{0}-s\right)^{2}} d s ; \\
\forall j=2: n, V_{j}\left(S_{j}\right) & =\int_{S_{j}^{*}}^{S_{j}} \frac{s-S_{j}^{*}}{S_{0}-s} d s .
\end{aligned}
$$

For all $j=1: n$, we can easily show that, $\forall S_{j} \in$ ] $0, S_{0}\left[\backslash\left\{S_{j}^{*}\right\}, V_{j}\left(S_{j}\right)>0\right.$, and $V_{j}\left(S_{j}\right)=0 \Leftrightarrow S_{j}=S_{j}^{*}$. After calculations, we obtain, $\forall j=2: n, \forall S \in \Omega$ and with $D_{j}=D_{j}^{*}:=\frac{Q_{j}^{*}}{\nu_{j}}$ :

$$
\begin{aligned}
\frac{d V_{j}}{d t}= & -\left(\mu\left(S_{j}\right)-\mu\left(S_{j}^{*}\right)\right)\left(S_{j}-S_{j}^{*}\right) \\
& -\frac{D_{j}^{*}\left(S_{0}-S_{j-1}^{*}\right)}{\left(S_{0}-S_{j}\right)\left(S_{0}-S_{j}^{*}\right)}\left(S_{j}-S_{j}^{*}\right)^{2} \\
& +\frac{D_{j}^{*}}{S_{0}-S_{j}}\left(S_{j-1}-S_{j-1}^{*}\right)\left(S_{j}-S_{j}^{*}\right), \\
\text { and } \frac{d V_{1}}{d t}= & -\left(\mu\left(S_{1}\right)-\mu\left(S_{1}^{*}\right)\right)\left(S_{1}-S_{1}^{*}\right) \frac{1}{S_{0}-S_{1}} .
\end{aligned}
$$

By use of the classical inequality verified for any $a, b, \gamma$ : $a b<\frac{1}{2}\left(\gamma^{2} a^{2}+\frac{1}{\gamma^{2}} b^{2}\right)$, and because $D_{j}^{*}<\frac{\nu_{j-1}}{\nu_{j}} D_{j-1}^{*}$ and $S_{j}<S_{j-1}$ which implies $\frac{1}{S_{0}-S_{j}}<\frac{1}{S_{0}-S_{j-1}}$, we then get, for any $\gamma_{j}, j=1: n$ :

$$
\begin{aligned}
\frac{d V_{j}}{d t} \leqslant & \frac{D_{j}^{*}}{S_{0}-S_{j}}\left(\frac{1}{2} \frac{1}{\gamma_{j}^{2}}-\frac{S_{0}-S_{j-1}^{*}}{S_{0}-S_{j}^{*}}\right)\left(S_{j}-S_{j}^{*}\right)^{2} \\
& -\left(\mu\left(S_{j}\right)-\mu\left(S_{j}^{*}\right)\right)\left(S_{j}-S_{j}^{*}\right) \\
& +\frac{\gamma_{j}^{2} \frac{\nu_{j-1}}{\nu_{j}} D_{j-1}^{*}}{2\left(S_{0}-S_{j-1}\right)}\left(S_{j-1}-S_{j-1}^{*}\right)^{2} .
\end{aligned}
$$

Consider now the function $V(S)=\sum_{j=1}^{n} \alpha_{j} V_{j}\left(S_{j}\right)$ with $\alpha_{j}>0$. We have:

$$
\begin{aligned}
& \frac{d V}{d t} \leqslant\left[\frac{\alpha_{2} \gamma_{2}^{2} D_{2}^{*}}{2}\left(S_{1}-S_{1}^{*}\right)-\alpha_{1}\left(\mu\left(S_{1}\right)-\mu\left(S_{1}^{*}\right)\right)\right] \frac{S_{1}-S_{1}^{*}}{S_{0}-S_{1}} \\
& -\sum_{j=2}^{n} \alpha_{j} \underbrace{\left(\mu\left(S_{j}\right)-\mu\left(S_{j}^{*}\right)\right)\left(S_{j}-S_{j}^{*}\right)}_{>0 \text { because } \mu \text { is increasing }} \\
& +\sum_{j=2}^{n-1}\left[\alpha_{j}\left(\frac{1}{2 \gamma_{j}^{2}}-\frac{S_{0}-S_{j-1}^{*}}{S_{0}-S_{j}^{*}}\right)+\frac{\alpha_{j+1} \gamma_{j+1}^{2} \nu_{j}}{2 \nu_{j+1}}\right] \frac{D_{j}^{*}\left(S_{j}-S_{j}^{*}\right)^{2}}{S_{0}-S_{j}} \\
& +\frac{\alpha_{n} D_{n}^{*}}{S_{0}-S_{n}}\left(\frac{1}{2} \frac{1}{\gamma_{n}^{2}}-\frac{S_{0}-S_{n-1}^{*}}{S_{0}-S_{n}^{*}}\right)\left(S_{n}-S_{n}^{*}\right)^{2}
\end{aligned}
$$


Let us consider the following parameters $\gamma_{j}, j=2: n$ :

$$
\gamma_{j}^{2}=\frac{S_{0}-S_{j}^{*}}{S_{0}-S_{j-1}^{*}}, \forall j=2: n,
$$

and $\alpha_{j}, j=1: n$, with $A_{j}, j=2: n$ some given positive constants:

$\alpha_{n}=2 A_{n} \frac{S_{0}-S_{n}^{*}}{S_{0}-S_{n-1}^{*}} \frac{\nu_{n} S_{0}}{Q_{m}}$,

$\alpha_{i}=\left(2 A_{j} \frac{\nu_{j} S_{0}}{Q_{m}}+\frac{\gamma_{j+1}^{2} \alpha_{j+1} \nu_{j}}{\nu_{j+1}}\right) \frac{S_{0}-S_{j}^{*}}{S_{0}-S_{j-1}^{*}}, j=2: n$,

$\alpha_{1}=\left(A_{1} S_{0}+\frac{\alpha_{2} \gamma_{2}^{2} Q_{M}}{2 \nu_{2}}\right) \frac{\left(a+S_{0}\right)\left(a+S_{1}^{*}\right)}{m a}$

We then have, $\forall j=2: n$ :

$$
\begin{array}{r}
\frac{D_{j}^{*}}{S_{0}-S_{j}}\left[\alpha_{j}\left(\frac{1}{2} \frac{1}{\gamma_{j}^{2}}-\frac{S_{0}-S_{j-1}^{*}}{S_{0}-S_{j}^{*}}\right)+\frac{\alpha_{j+1} \gamma_{j+1}^{2} \nu_{j}}{2 \nu_{j+1}}\right] \leqslant-A_{j}, \\
{\left[\frac{\alpha_{2} \gamma_{2}^{2} D_{2}^{*}}{2}-\alpha_{1} \frac{\left(\mu\left(S_{1}\right)-\mu\left(S_{1}^{*}\right)\right)}{S_{1}-S_{1}^{*}}\right] \frac{1}{S_{0}-S_{1}} \leqslant-A_{1},} \\
\text { and } \frac{\alpha_{n} D_{n}^{*}}{S_{0}-S_{n}}\left(\frac{1}{2} \frac{1}{\gamma_{n}^{2}}-\frac{S_{0}-S_{n-1}^{*}}{S_{0}-S_{n}^{*}}\right) \leqslant-A_{n} .
\end{array}
$$

So, finally, we have:

$$
\frac{d V}{d t} \leqslant-\sum_{j=2}^{n} \alpha_{j}\left(\mu\left(S_{j}\right)-\mu\left(S_{j}^{*}\right)\right)\left(S_{j}-S_{j}^{*}\right)-\sum_{j=1}^{n} A_{j}\left(S_{j}-S_{j}^{*}\right)^{2}
$$

that is, $\frac{d V}{d t} \leqslant 0, \forall S \in \Omega$, and:

$$
\left\{S \in \Omega \text { such that } \frac{d V}{d t}=0\right\}=\left\{S^{*}\right\} .
$$

\subsection{Control design}

The control law is obtained by application of proposition 1 to the system (24) with $\Omega$ given by (25) and $V$ defined by:

$$
V(S)=\alpha_{1} \int_{S_{1}^{*}}^{S_{1}} \frac{s-S_{1}^{*}}{\left(S_{0}-s\right)^{2}} d s+\sum_{j=2}^{n} \alpha_{j} \int_{S_{j}^{*}}^{S_{j}} \frac{s-S_{j}^{*}}{S_{0}-s} d s
$$

This control law ensures the asymptotic stability of the closed-loop system, the convergence of $S$ to the setpoint $S^{*}$ (without the knowledge of the steady state constant input values $Q_{j}^{*}$ ) and the fulfillment of the constraint (18) all along the trajectories. It is expressed by:

$$
Q_{j}=\left(Q_{M}-Q_{m}\right) \prod_{k=1}^{j} \theta_{k}+Q_{m}
$$

with $\sigma_{j}\left(\theta_{j}\right)=k_{j}\left(\theta_{j}-1\right) \theta_{j}, \theta_{j}(0) \in(0,1), k_{j}>0$ and:

$$
\begin{aligned}
& \frac{d \theta_{n}}{d t}=\sigma_{n}\left(\theta_{n}\right) \frac{\partial V}{\partial x_{n}} \psi_{n}(x), \\
& \frac{d \theta_{j \neq n}}{d t}=\sigma_{j}\left(\theta_{j}\right)\left[\frac{\partial V}{\partial x_{j}} \psi_{j}(x)+\sum_{l=j+1}^{n} \frac{\partial V}{\partial x_{l}} \psi_{l}(x) \prod_{k=j+1}^{l} \theta_{k}\right],
\end{aligned}
$$

with $\forall j=2: n$ :

$$
\begin{aligned}
& \frac{\partial V}{\partial S_{j}} \psi_{j}(S)=\alpha_{j} \frac{Q_{M}-Q_{m}}{\nu_{j}} \frac{\left(S_{j-1}-S_{j}\right)\left(S_{j}-S_{j}^{*}\right)}{S_{0}-S_{j}} \\
& \text { and } \frac{\partial V}{\partial S_{1}} \psi_{1}(S)=\alpha_{1} \frac{Q_{M}-Q_{m}}{\nu_{1}} \frac{S_{1}-S_{1}^{*}}{S_{0}-S_{1}}
\end{aligned}
$$

\subsection{Numerical simulation}

\begin{tabular}{|c|c|c|c|c|}
\hline \multirow{2}{*}{$\begin{array}{l}\text { reactor } \\
\text { number }\end{array}$} & \multirow{2}{*}{$\begin{array}{l}\text { Volume } \\
{[L]}\end{array}$} & \multicolumn{2}{|c|}{ Input flow rate $\left[L \cdot h^{-1}\right.$} & \multirow{2}{*}{$\begin{array}{c}\text { Set-point } \\
{\left[g \cdot L^{-1}\right]}\end{array}$} \\
\hline & & Initial & Steady state & \\
\hline$i$ & $\nu_{i}$ & $Q_{i}^{0}$ & $Q_{i}^{*}$ & $S_{i}^{*}$ \\
\hline 1 & 1.0 & 0.230 & 0.235 & 0.3339 \\
\hline 2 & 0.7 & 0.225 & 0.150 & 0.1768 \\
\hline 3 & 0.5 & 0.130 & 0.125 & 0.0840 \\
\hline 4 & 0.7 & 0.050 & 0.120 & 0.0286 \\
\hline
\end{tabular}

For the numerical simulation, we have considered a series of $n=4$ reactors, as in the multi-stage continuous fermentor presented in $[10,11]$. The values of the parameters of the model are given here after:

$$
\begin{array}{r}
k=0.0606[-] ; a=1.57\left[\mathrm{~g} \cdot \mathrm{L}^{-1}\right] ; m=1.34[0.9756] ; \\
X_{0}=0\left[\mathrm{~g} \cdot \mathrm{L}^{-1}\right] ; S_{0}=0.425\left[\mathrm{~g} \cdot \mathrm{L}^{-1}\right] .
\end{array}
$$

These are the same values as the ones considered in [11] for the modeling of the growth of the yeast on the nitrogen during the wine fermentation.

The values of the volumes, of the initial and steady state values of the input flow rates and of the chosen set-point are given in table 1 .

Table 1: Volumes of the reactors, initial and steady-state values of the input flow rates and substrate concentration set-point of the numerical simulation.

Finally, the values of the parameters of the control law are given here-after:

$$
\begin{array}{r}
k_{1}=510^{-4} ; k_{2}=510^{-3} ; k_{3}=210^{-1} ; k_{4}=5 ; \\
Q_{M}=0.25\left[L . h^{-1}\right] ; Q_{m}=0.01\left[L \cdot h^{-1}\right] ; \\
\text { and } A_{i}=4, \forall i=1: 4 .
\end{array}
$$

For the numerical implementation of the control law, the modification used in [1] has been applied, which leads to the following practical control law:

$$
\frac{d \theta_{j}}{d t}= \begin{cases}\max (0, C(\theta)) & \text { if } \theta_{j} \in\left[v_{j}, v_{j}+\varepsilon[\right. \\ C(\theta) & \text { if } \theta_{j} \in\left[\overline{v_{j}}+\varepsilon, \overline{v_{j}}-\varepsilon\right] \\ \min (0, C(\theta)) & \text { if } \left.\left.\theta_{j} \in\right] \overline{\overline{v_{j}}}-\varepsilon, \overline{v_{j}}\right]\end{cases}
$$

where $C(\theta)$ is the expression given in (33). The parameter $\varepsilon$ has been chosen equal to 0.04 .

The trajectories of the closed loop system (24-9) are shown in figure 1 . We can see that the substrate concentration in each of the 4 reactors reaches its set-point value, and that the control inputs $Q_{i}$ fulfill the saturation constraint (18) all along the trajectories. 

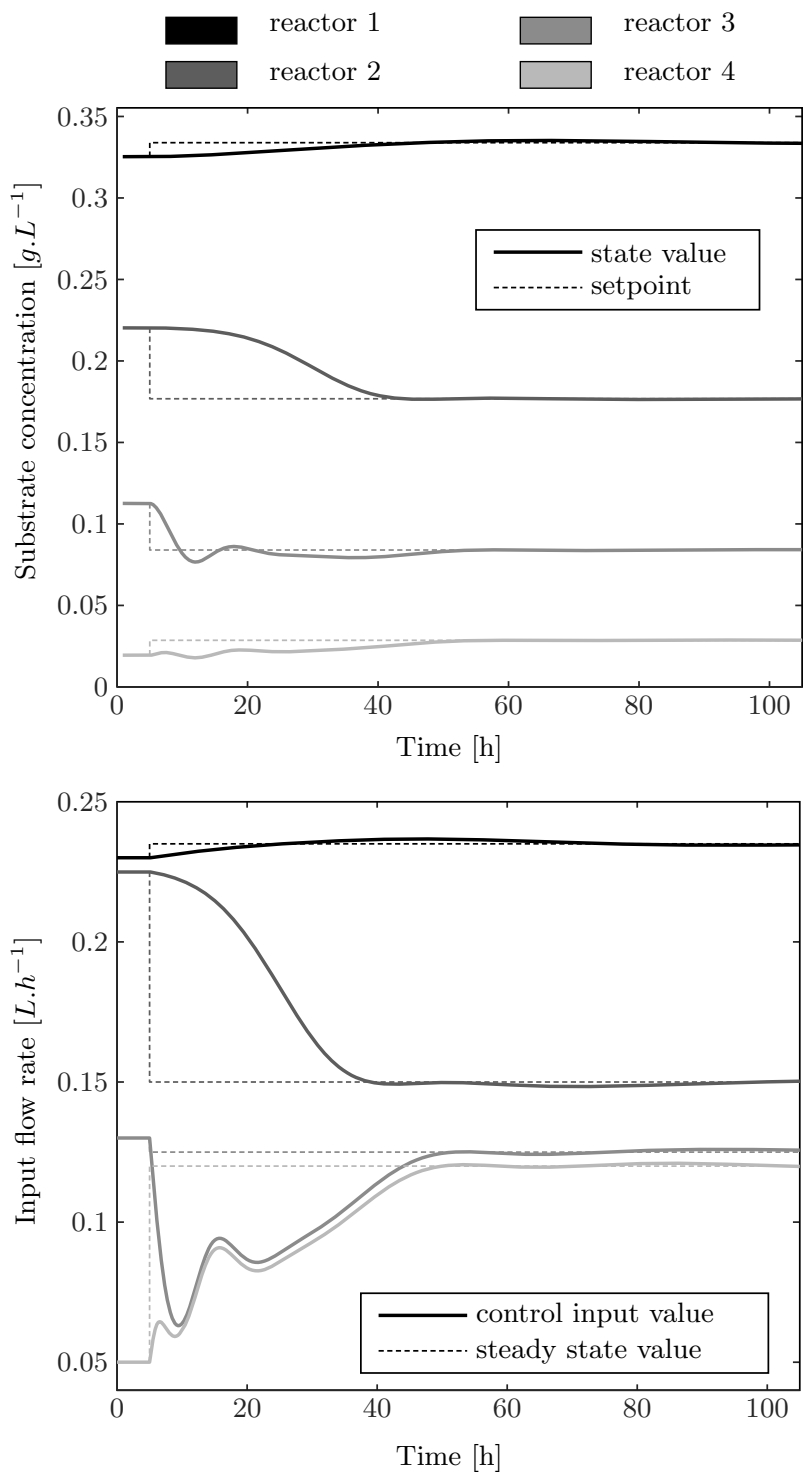

Figure 1: Validation of the control strategy on an example of a nonlinear cascade systems composed of 4 bioreactors interconnected in series.

\section{CONCLUSION}

In this paper, the result that A.Antonelli and A.Astolfi have presented in [1] has been extended to a class of multi-input nonlinear systems. This result has then been used for the control of a class of nonlinear cascade systems with input-dependent saturation. The particularity of these systems comes from the form of the saturation which is a constraint of "order": the control input of one subsystem has to be smaller than the control input of the preceding subsystem of the cascade structure. The design of the control law has been applied on the concrete example of a series of interconnected continuous reactors. The control law has then been validated on numerical simulations and gives promising results. Nevertheless, a lot of work still remain to do. In particular, the choice of the parameters values for the control law has to be studied. Indeed, even if the parameters $A_{i}$ are directly linked to the convergence speed of the state to the set-point, the parameters $k_{i}$ also have effect on the closed loop behavior. The extension of the result to the case of more general forms of interconnected systems is also an interesting problem which will be addressed in the future.

\section{REFERENCES}

[1] R. Antonelli and A. Astolfi, "Continuous stirred tank reactors: easy to stabilise?," Automatica, vol. 39, no. 10, pp. 1817-1827, 2003.

[2] S. Tarbouriech and M. Turner, "Anti-windup design: an overview of some recent advances and open problems," IET control theory $\& 3$ applications, vol. 3, no. 1, pp. 1-19, 2009.

[3] S. Bezzaoucha, B. Marx, D. Maquin, and J. Ragot, "Stabilization of nonlinear systems subject to actuator saturation," in Fuzzy Systems (FUZZ), 2013 IEEE International Conference on, pp. 1-6, IEEE, 2013.

[4] T. A. Kendi and F. J. Doyle III, "An anti-windup scheme for multivariable nonlinear systems," Journal of process control, vol. 7, no. 5, pp. 329-343, 1997.

[5] F. J. Doyle III, "An anti-windup input-output linearization scheme for SISO systems," Journal of process control, vol. 9, pp. 213-220, 1999.

[6] Q. Hu and G. Rangaiah, "Anti-windup schemes for uncertain nonlinear systems," IEE Proceedings - Control Theory and Applications, vol. 147, no. 3, p. 321, 2000.

[7] S.-S. Yoon, J.-K. Park, and T.-W. Yoon, "Dynamic anti-windup scheme for feedback linearizable nonlinear control systems with saturating inputs," Automatica, vol. 44, pp. 3176-3180, Dec. 2008.

[8] R. Antonelli, J. Harmand, J.-P. Steyer, and A. Astolfi, "Set-point regulation of an anaerobic digestion process with bounded output feedback," Control Systems Technology, IEEE Transactions on, vol. 11, no. 4, pp. 495-504, 2003.

[9] G. Escobar, D. Chevreau, R. Ortega, and E. Mendes, "An adaptive passivity-based controller for a unity power factor rectifier," Control Systems Technology, IEEE Transactions on, vol. 9, no. 4, pp. 637-644, 2001.

[10] T. Clement, M. Perez, J. Mouret, J. Sablayrolles, and C. Camarasa, "Use of a continuous multistage bioreactor to mimic winemaking fermentation," International journal of food microbiology, vol. 150, no. 1, pp. 42-49, 2011.

[11] C. Casenave, D. Dochain, J. Harmand, M. Perez, A. Rapaport, and J.-M. Sablayrolles, "Control of a multi-stage continuous fermentor for the study of the wine fermentation," in 19th IFAC World Congress 2014, Cape Town (South Africa), pp. 6192-6197, August 24-29 2014. 